\title{
Uterine Prolapse: The Other Exceptional Complication of Spina Bifida in Newborns
}

\author{
T. Mukenge1, F. B. Balde ${ }^{1 *}$, Z. Benmassaoud1, I. Oualili1', O. Alaoui1,2, A. Mahmoudi1,2, \\ K. Khattala1,2, Y. Bouabdallah1,2 \\ ${ }^{1}$ Department of Pediatric Surgery, Hassan II University Hospital, Fez, Morocco \\ ${ }^{2}$ Sidi Mohamed Ben Abdellah University, Fez, Morocco \\ Email: ^fatoumatabinta.balde@usmba.ac.ma
}

How to cite this paper: Mukenge, T., Balde, F.B., Benmassaoud, Z., Oualili, I., Alaoui, O., Mahmoudi, A., Khattala, K. and Bouabdallah, Y. (2021) Uterine Prolapse: The Other Exceptional Complication of Spina Bifida in Newborns. Open Journal of Pediatrics, 11, 50-54.

https://doi.org/10.4236/ojped.2021.111005

Received: January 9, 2021

Accepted: March 5, 2021

Published: March 8, 2021

Copyright $\odot 2021$ by author(s) and Scientific Research Publishing Inc. This work is licensed under the Creative Commons Attribution International License (CC BY 4.0).

http://creativecommons.org/licenses/by/4.0/

\begin{abstract}
Introduction: Spina bifida is the most common neural tube defect. Uterine prolapse is an exceptional presentation of its complications. We aim to describe the clinical and progressive features of uterine prolapse in a newborn baby with spina bifida. Observation: 19-day-old newborn. Admitted for spina bifida. The clinical examination showed an anal gaping, the presence of uterovaginal prolapse and bilateral equine varus clubfoot. The particularity was that this prolapse had a spontaneous resolution but appears with screams. We made a compression bandage at the time of resolution. After a 6-month of follow-up, the baby is in good general condition and, there is no recurrence of the prolapse. Conclusion: Uterine prolapse is a rare complication of spina bifida. The main therapeutic component remains the prevention of spina bifida.
\end{abstract}

\section{Keywords}

Uterovaginal Prolapse, Congenital, Spina Bifida

\section{Introduction}

Spina bifida is a neural tube closure defect occurring during the fourth week of gestation. It is the most common neural tube defect [1]. It has consequences whose physiopathological evolution is often severe and requires long and evolutionary management. These are hydrocephalus, sensory and motor deficits, orthopaedic and urogenital abnormalities. The uterine prolapse is an exceptional presentation of the long list of its complications. In the neonatal period, uterine prolapse is associated with spina bifida in more than $80 \%$ [2] [3]. Management ranges from manual reduction to surgical treatment whose radicality has evolved 
over the years [4]. The purpose of this study is to describe the clinical and evolutionary characteristics of the case of a newborn with uterine prolapse in spina bifida.

\section{Observation}

Infant, 19 days old, female, from a non-consanguine marriage, mother, 18 years old G1P1 no folic acid intake by the mother, irregular follow-up pregnancy. No antenatal imaging. Born by natural way, to term according to the date of the last menses with good adaptation to the extra-uterine life. Admitted for a lumbosacral swelling seen since birth. She was in good general condition, stable and apyretic. There was a $4 \times 4 \mathrm{~cm}$ lumbosacral myelomeningocele (Figure 1). An anal gaping, a prolapsed mass between the two large lips (uterine prolapsus) (Figure 2). According to the mother, this mass is spontaneously resolute but reappears after an episode of crying. The urethral orifice was normal. The baby also had a bilateral equine varus clubfoot (EVCF). The weight was $3 \mathrm{~kg} 200$, and the head perimeter was $34 \mathrm{~cm}$. We performed a compression dressing at the time of spontaneous resolution of the prolapse for 24 hours. We noted a complete disappearance of the prolapse, even at the scrams. We cure the myelomeningocele by closing the neural plaque, closure of the dura mater and cutaneous closure. We noted an increase of $2 \mathrm{~cm}$ of the head perimeter 6-days after surgery of spina bifida. Postoperative trans-fontanelle ultrasound at $J+6$ found hydrocephalus. We performed a ventriculoperitoneal shunt (VPS) as indicated by our protocol for all active hydrocephalus. Ponsetti's protocol was started for the bilateral EVCF.

After 6-months of follow-up, the baby is in good general condition, no recurrence of the prolapse (Figure 3). However, there is a delay in motor vehicle acquisitions: head holding and sitting position.

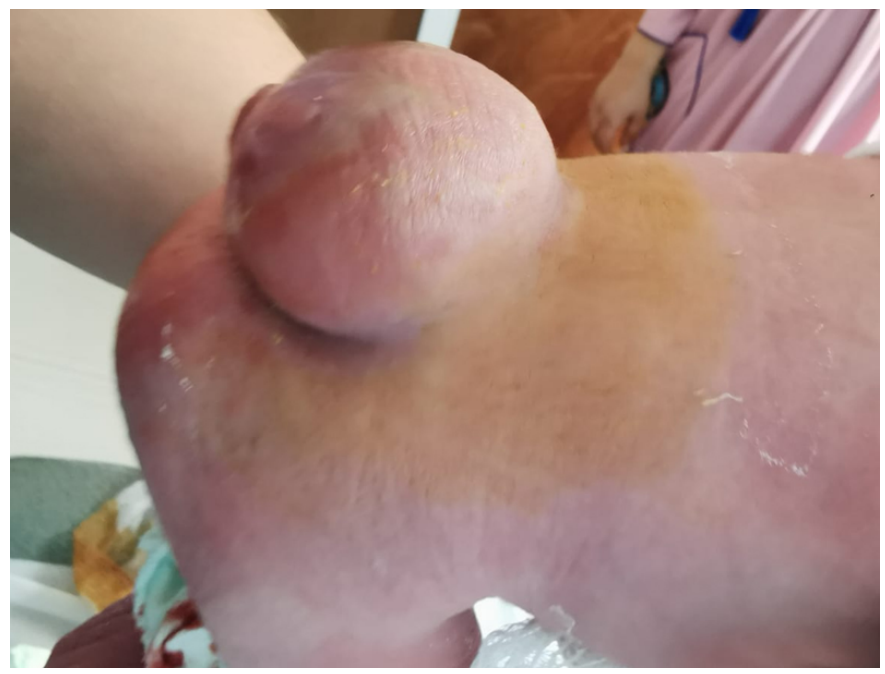

Figure 1. Profile image of the baby's back, a $4 \times 4 \mathrm{~cm}$ lumbosacral spina bifida covered at the periphery by the skin, an unbroken translucent membrane un the centre. 


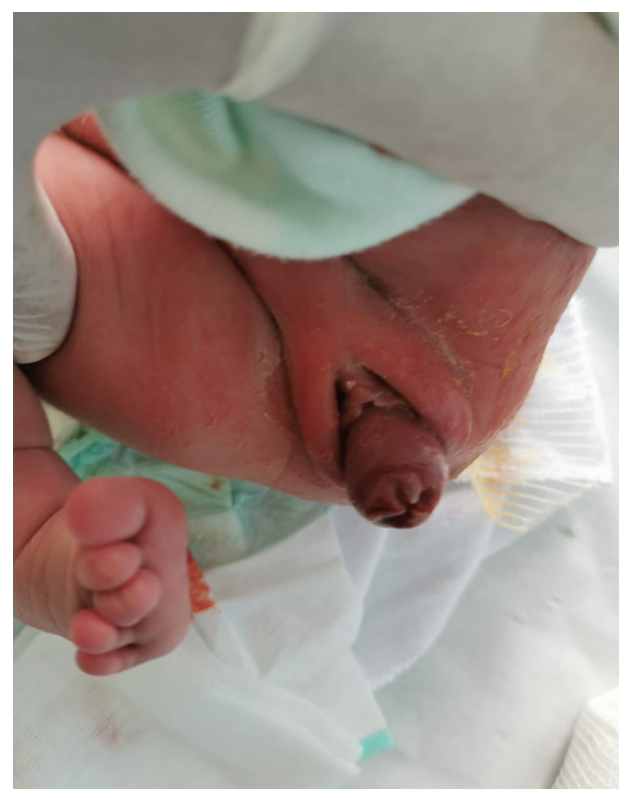

Figure 2. Clinical image showing uterine prolapse of the newborn.

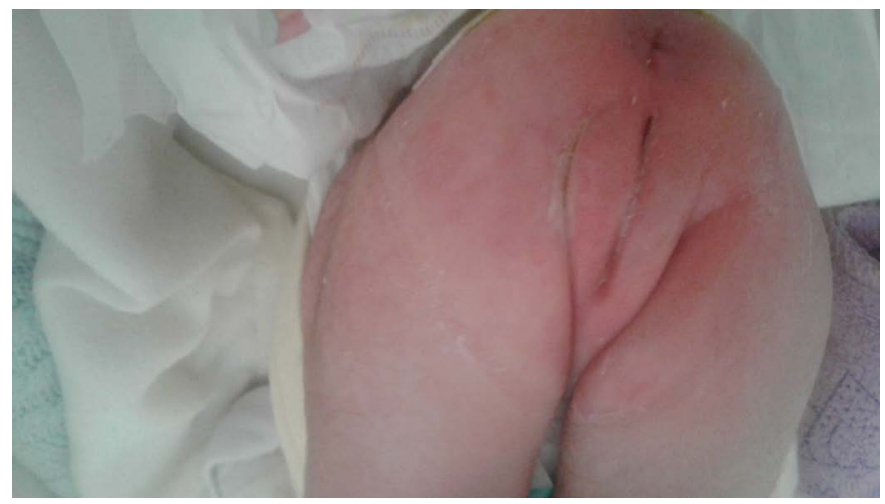

Figure 3. The clinical image of the 3-month follow-up showing an absence of uterine prolapse.

\section{Discussion}

Uterine prolapse is an unfolding circumferential protrusion to a varying degree of the uterus through the vaginal opening. Caused by the absence of innervation of the muscular structures of the perineum. In particular, the levator anus, which is the first support for the pelvic organs [2].

The neonatal forms are exceptional. The first description was in 1723, and since less than 100 cases have been reported in the literature [5]. This case is the second encountered in our training in 10 years of experience. In the neonatal period, uterine prolapse is more than $80 \%$ associated with spina bifida [2].

It is essential to formally eliminate the other diagnostic possibilities, in front of an inter labial mass of the newborn: such as the vaginal polyp, urethral prolapse, a paraurethral cyst, or rhabdomyosarcoma [2]. In severe forms, there it is associated with rectal prolapse [5].

Uterine prolapse is a therapeutic emergency to prevent complications. Such as 
urinary obstruction, which can lead to hydronephrosis, or endometrial metaplasia [6] [7]. No consensus management protocol has been established. The authors make proposals for support through the sharing of experiences. It ranges from conservative treatment to surgical treatment. Manual reduction under anaesthesia has been strongly reported [1] [6].

This reduction is held in place either by an intravaginal wick with a compression bandage for 72 hours on average [6]. Using a Folley catheter with the inflated balloon held in place for 2-weeks helps keep the reduction [2] [3].

Frequently the procedure is repeated because of recurrence of the prolapse, or require surgical treatment. Surgical treatment ranges from a simple labial suture to amputation of the cervix or even hysterectomy. Transabdominal uteropexy has also been reported [1] [3].

Cases of spontaneous resolution are reported with isolated uterine prolapse of the newborn [3].

The peculiarity is that our patient presented a spontaneous reduction in prolapse, which is why we took this advantage to apply a compression bandage held in place for 24 hours. It saved the baby from the risks of iterative anaesthesia.

The other possibility was to cure the myelomeningocele and reduce the prolapse at the same time. But the prolapse had completely disappeared.

Hydrocephalus can depend or not to the spina bifida. Its can appear spontaneously or after cure of myelomeningocele. Anywhere discussion is open about when to conduced a ventriculoperitoneal shunt. From 1998 to 2014, hydrocephalus treatment has become more delayed. A meta-analysis found that there is not differ between delayed and simultaneous hydrocephalus treatment [8].

After a follow-up of 6 months, no recurrence of the prolapse was reported even in the literature, no case of recurrence is reported

\section{Conclusion}

Uterine prolapse is an exceptional complication of spina bifida. The treatment ranges from manual reduction to actual surgery, the radical nature of which has changed over the years. In all cases, the therapeutic component remains the prevention of Spina bifid by taking folic acid before and during the first months of pregnancy.

\section{Ethical Aspect}

Parental consent has obtained for the use of patient data, and no image allows identification.

\section{Conflicts of Interest}

The authors declare no conflicts of interest regarding the publication of this paper.

\section{References}

[1] Aykanat, A., Solakoglu, E., Bilginer, B., Celik, H.T. and Yigit, S. (2020) Uterovaginal 
Prolapse in a Newborn with Meningomyelocele: Case Report. Journal of Pediatric and Adolescent Gynecology, 33, e607-e609.

https://doi.org/10.1016/j.jpag.2020.04.001

[2] Abdelsalam, S.E.A., Desouki, N.M. and Alaal, N.A.A. (2006) Use of Foley Catheter for Management of Neonatal Genital Prolapse: Case Report and Review of the Literature. Journal of Pediatric Surgery, 41, 449-452.

https://doi.org/10.1016/j.jpedsurg.2005.11.031

[3] Baskaran, D. and Mohan Nazeeb, P. (2012) Purse String Suturing in a Neonatal Prolapsed Uterus. Indian Journal of Surgery, 74, 143-145.

https://doi.org/10.1007/s12262-011-0361-Z

[4] Ajabor, L.N. and Okojie, S.E. (1976) Genital Prolapse in the Newborn. International Surgery, 61, 496-497.

[5] Yıldızdaş, H.Y., Ece, Ü., Yurdakul, G.B. and Kolağasıgil, E. (2019) Spontaneously Resolved Uterine Prolapse in a Neonate with Spina Bifida. The Turkish Journal of Pediatrics, 61, 979-981. https://doi.org/10.24953/turkjped.2019.06.026

[6] Jijo, Z.W., Betele, M.T. and Ali, A.S. (2018) Congenital Uterovaginal Prolapse in a Newborn. Case Reports in Obstetrics and Gynecology, 2018, Article ID: 1425953. https://doi.org/10.1155/2018/1425953

[7] Lockwood, G., Durkee, C. and Groth, T. (2012) Genital Prolapse Causing Urinary Obstruction and Hydronephrosis in a Neonate: A Case and Review of the Literature. Journal of Neonatal Surgery, 1, 39.

[8] McCarty, D.J., Sheinberg, D.L., Luther, E. and McCrea, H.J. (2019) Myelomeningocele Associated Hydrocephalus: Nationwide Analysis and Systematic Review. Journal of Neurosurgery, 47, E5. https://doi.org/10.3171/2019.7.FOCUS19469 